\title{
The RKKY Coupling of Two Magnetically Doped Monolayers in Thin Films
}

\author{
K. Szalowski* AND T. BalCERZAK \\ Department of Solid State Physics, University of Łódź \\ Pomorska 149/153, 90-236 Łódź, Poland
}

The Gauss-type exchange contact potential is applied to derive the Ruderman-Kittel-Kasuya-Yosida interaction in thin films. The interlayer magnetic coupling energies and the critical Curie/Néel temperatures are calculated. The quantum size effects are found to cause long-range oscillatory behavior of these quantities vs. film thickness, with the period much longer than the usual RKKY characteristic oscillations.

PACS numbers: 75.30.-m, 75.50.-y, 75.70.-i

\section{Introduction}

In the studies of interaction between localized magnetic moments and charge carriers usually a contact form of the exchange potential is assumed. However, as the localized magnetic moments originate from the inner electronic orbitals (e.g. of $d$-type in diluted magnetic semiconductors), they manifest a finite spatial extension [1]. Consequently, it seems necessary to introduce a "diffused" exchange potential. This, in turn, modifies the perturbative RKKY-type interaction between the localized spins. Such an effect for the bulk case was considered so far in Refs. $[2,3]$ but, as far as we know, not yet in thin films.

In this work the Gaussian distribution for the exchange coupling potential is applied, in order to describe the RKKY mechanism of localized spins interaction in ultra-thin films modeled by an infinite quantum well $[4,5]$. A model diluted system based on the fcc structure with surface orientation (001) is considered, with only two monolayers inside the film containing magnetic ions. Such a choice reflects the geometry of experimentally investigated structures of magnetically-doped semiconductors, such as $\mathrm{Ga}_{1-x} \mathrm{Mn}_{x}$ As. The model is parameterized by the total thickness of the film, the distance between the magnetic monolayers,

*corresponding author; e-mail: kszalowski@uni.lodz.pl 
"diffusion" of the contact potential as well as the concentration of the free charge carriers.

The finite width of the contact potential removes an unphysical divergence of the RKKY exchange integral in the direction perpendicular to the film plane, and allows the interaction energy of two monolayers to be studied. The present study comprises the coupling energy calculation of two magnetic monolayers as a function of their separation, the film thickness and charge concentration. The ferromagnetic or antiferromagnetic orientation of magnetizations in these monolayers is considered. In the molecular field approximation (MFA) the critical (Curie/Néel) temperatures are also calculated.

\section{Theory}

The model thin film is considered as a set of $n$ monoatomic planes, each having its own thickness $d$. Thus, the total thickness of the film is given by $n d$. In the simplest approach, when the film is situated in the vacuum, we assume that it forms a one-dimensional (1D) quantum well of the infinite depth. For such a system the RKKY exchange integral has been derived within the free electron approximation $[5,6]$. In the derivation the perturbation calculus for the point-like contact potential between localized spin $S_{\nu i}^{z}$ and the charge carrier spin $s^{z}(\boldsymbol{R})$ has been used. Index $\nu i$ denotes the position of the localized spin, i.e., $\nu$ is the number of monolayer $(\nu=1,2, \ldots, n)$ and $i$ indicates the position within the plane.

The perturbation Hamiltonian has a form

$$
\widetilde{\mathcal{H}}_{\nu i}=-A p(\boldsymbol{R}) S_{\nu i}^{z} s^{z}(\boldsymbol{R}),
$$

where $\nu i$ can be chosen as the center of the coordination system for the random vector $\boldsymbol{R}=\boldsymbol{r}+z \hat{z}$. We assume that $\boldsymbol{r}$ lies in the film plane and the $\hat{z}$-direction is perpendicular to the film. $A$ constant in (1) is connected with the exchange interaction of $z$-components of both spins.

For the point-like contact potential in the form of the Dirac $\delta$-function, $p(\boldsymbol{R})=\delta(\boldsymbol{R})$, the perturbation Hamiltonian has previously been utilized for derivation of the RKKY exchange interaction in 1D, 2D, and 3D systems [7-11]. In the present paper we assume that the probability density distribution for the localized spin is given by the Gaussian function

$$
p(\boldsymbol{R})=\frac{1}{\sigma^{3}(2 \pi)^{3 / 2}} \mathrm{e}^{-R^{2} /\left(2 \sigma^{2}\right)},
$$

instead of Dirac's $\delta$-distribution. By the same token, the analogous procedure as in [5] yields the effective interaction Hamiltonian for the spin-pair $\left(S_{\nu i}^{z}, S_{\mu j}^{z}\right)$ in the form

$$
\mathcal{H}_{\nu i, \mu j}=-J(r, z) S_{\nu i}^{z} S_{\mu j}^{z} .
$$

The RKKY exchange integral, $J(r, z)$, for a spin-pair separated by the distance $r$ in the plane parallel to the film and by $z$ in the direction perpendicular to the surface, can be expressed by the final formula 


$$
\begin{gathered}
J(r, z)=C\left(\frac{a}{d}\right)^{4} \frac{\pi}{4 n^{2}} \int_{0}^{\infty} \mathrm{d} y\left[y J_{0}\left(y \frac{r}{d}\right) \mathrm{e}^{-(\sigma / d)^{2} y^{2}}\right. \\
\left.\times \sum_{p=-\infty}^{\infty} \frac{\chi_{p, y}}{\chi_{0}} \cos \left(\frac{2 \pi}{n} \frac{z}{d} p\right) \mathrm{e}^{-(\sigma / d)^{2}(2 \pi p / n)^{2}}\right] .
\end{gathered}
$$

The energy constant $C$ amounts to $C=2 m A^{2} / \pi h^{2} a^{4}$, where $a$ is the lattice constant. $J_{0}(u)$ is the Bessel function of the 1st order and is given by $\pi J_{0}(u)=$ $\int_{0}^{\pi} \mathrm{d} t \cos (u \cos t)$. In Eq. (4) $\chi_{p, y}$ denotes the paramagnetic susceptibility of the charge carriers in thin film, whose explicit form has been derived in [12]. In order to avoid a redundant repetition of the formalism this susceptibility will not be characterized here. It contains the dependence on the (dimensionless) charge carriers concentration $\rho=n_{\mathrm{e}} d^{3}$, where $n_{\mathrm{e}}$ is the number of charge carriers per unit volume of the system. $\chi_{0}$ in the formula (4) denotes the Pauli paramagnetic susceptibility of $2 \mathrm{D}$ system per unit area, i.e., $\chi_{0}=m\left(g \mu_{\mathrm{B}}\right)^{2} / 4 \pi \hbar^{2}$. In the particular case of point-like contact potential (when $\sigma \rightarrow 0$ ) the formula (4) reproduces the result obtained in $[5,6]$. On the other hand, for $n \rightarrow \infty$ the result is convergent to the bulk $3 \mathrm{D}$ case.

Equation (4) is suitable for numerical calculations of the RKKY exchange integral in thin films with an arbitrary oriented spin-pair, for which the relative position of spins is defined by the distances $(r, z)$. On this basis, depending on the standard deviation $\sigma$ of the contact potential, various thermodynamic properties of the thin film can be obtained. In particular, for two magnetically-doped monolayers possessing the magnetic-ion concentration $x$ and separated by the distance $z$, the magnetic coupling energy can be calculated. It is assumed that the magnetization within each monolayer is uniform and that both planes are magnetically ordered. The effective magnetic coupling of both subsystems can either be negative (ferromagnetic) or positive (antiferromagnetic). In the ground state this interlayer magnetic coupling (IMC) energy per one lattice site is defined by:

$$
E_{\mathrm{IMC}}=-x^{2} S^{2} \sum_{k} z_{k}^{\prime} J\left(r_{k}^{\prime}, z\right)
$$

where $S$ is the spin magnitude. Symbol $z_{k}^{\prime}$ denotes the coordination number for the lattice sites belonging to the other monolayer and located on the $k$-th coordination zone with in-plane radius $r_{k}^{\prime}$.

Apart from the interlayer coupling, by taking into account the energy of interaction among spins from the same layer $(z=0)$, whose geometrical distribution is determined by the coordination numbers $z_{k}$ and the zone radii $r_{k}$, the full magnetic energy is obtained. This energy can be attributed to the critical (Curie/Néel) temperature of the system when the molecular field approximation is adopted. The critical temperature in MFA is given by the expression 


$$
k_{\mathrm{B}} T_{\mathrm{c}}=x \frac{S(S+1)}{3}\left[\sum_{k} z_{k} J\left(r_{k}, 0\right) \pm \sum_{k} z_{k}^{\prime} J\left(r_{k}^{\prime}, z\right)\right] .
$$

The solution with "+" corresponds to the Curie temperature and is valid for the ferromagnetic coupling of the monolayers $\left(E_{\mathrm{IMC}}<0\right)$, whereas the solution with "-" corresponds to the Néel temperature and is applicable to the antiferromagnetic coupling $\left(E_{\mathrm{IMC}}>0\right)$. The summation over the coordination zones $k$ in both monolayers is carried out until the satisfactory numerical convergence of energy is achieved.

\section{Numerical results and discussion}

The numerical results are obtained for the films with fcc structure, (001) surface orientation and different thicknesses $n$. In particular, when the interlayer distance $|\mu-\nu|=z / d$ is even, we have $r_{k}^{\prime}=r_{k}$ and $z_{k}^{\prime}=z_{k}$ for both monolayers. The "diffusion" parameter of the contact potential $\sigma / d=0.25$ is assumed.

In Fig. 1 the magnetic interlayer coupling energy $-E_{\mathrm{IMC}}$ is shown upon the charge carriers concentration $\rho$. For illustration, two thicknesses of the film have been selected: $n=6$ and $n=10$. The bulk case with $n \rightarrow \infty$ is also shown. The interlayer distance $z / d=4$ is constant for all three cases. It is seen in Fig. 1 that the charge carriers concentration influences the coupling energy, modifying not only its magnitude but also the sign. The positive (or negative) $-E_{\mathrm{IMC}}$ corresponds to the ferromagnetic (or antiferromagnetic) couplings. Taking into account that $z=$ const, the differences between various curves illustrate the size effects. The abrupt changes of $-E_{\mathrm{IMC}}$ vs. $\rho$ are the manifestation of the quantum size effects which are caused by discretization of the Fermi surface in thin films and analogous changes in the density of states at the Fermi level [12].

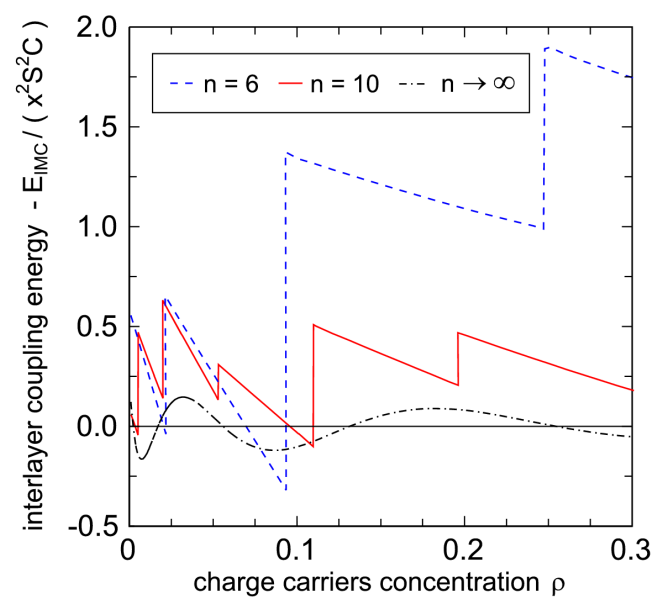

Fig. 1. The IMC energy for $z / d=4$ and different thicknesses $n$ upon the charge carriers concentration $\rho$. 


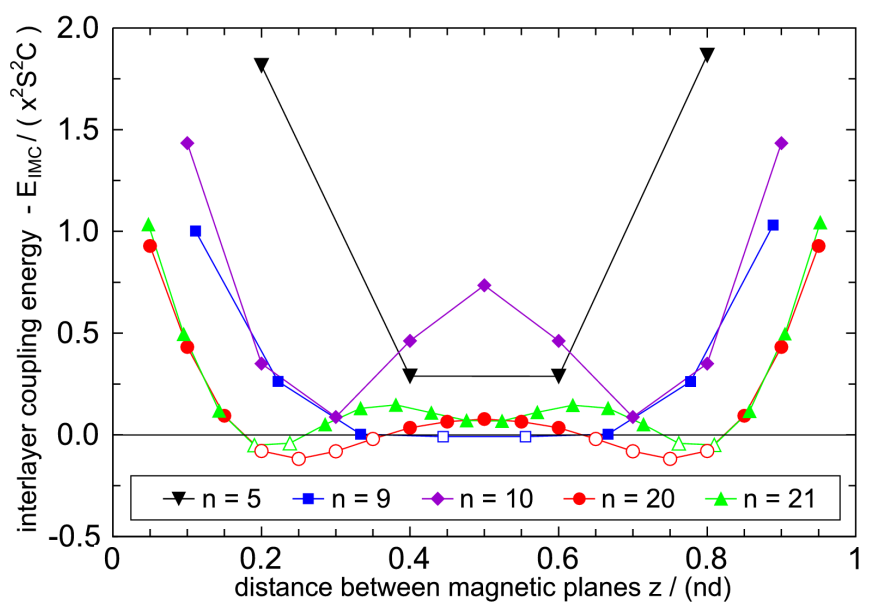

Fig. 2. Ferromagnetic and antiferromagnetic interlayer coupling energy vs. the distance between magnetic planes for several film thicknesses $n$.

In Fig. 2 the $-E_{\mathrm{IMC}}$ is presented as a function of normalized interlayer distance $z /(n d)$, when only two monolayers out of $n$ have been magnetically doped. In this case it is assumed that each impurity atom donates one free charge carrier to the conduction band and the impurity concentration is $x=0.05$, an assumption that gives $\rho=x / n$. Several curves for various thin film thicknesses $n$ are presented. A symmetrical enhancement of the energy coupling with respect to the distance $z$ corresponding to half-thickness of the film is seen. An enhancement of the RKKY interaction between the surface monolayers is characteristic of thin films [5]. The weakening of the interlayer coupling when $n$ increases is connected with decrease in the free-carriers concentration since only 2 monolayers have been doped in the whole system. The coupling energy presented in Fig. 2 remains mostly ferromagnetic, $-E_{\mathrm{IMC}}>0$ (which is marked by filled symbols in Fig. 2). However, at some distances $z$ where $-E_{\mathrm{IMC}}<0$ (empty symbols) the two monolayers are coupled antiferromagnetically. It is also worth noticing that for $n=10$ and $n=21$ the interlayer coupling becomes markedly enhanced and the shape of the curve changes. This behavior, being oscillatory in character when $n$ increases, is characteristic of the thin film and results from quantization of the Fermi surface.

In the last figure (Fig. 3) the Curie/Néel temperatures are presented for the same film parameters as in Fig. 2. The shape of the phase transition curves is somewhat similar to the IMC curves from Fig. 2. In particular, the analogous symmetry with respect to $z$-distance is visible. The Curie temperatures are marked by filled symbols, whereas the Néel temperatures represented by empty symbols are obtained for the antiferromagnetically coupled monolayers (i.e., when $E_{\mathrm{IMC}}>0$ ). Increasing the thickness from $n=9$ to $n=10$ and from $n=20$ to $n=21$ a change in the curve shape is observed together with some increase in the Curie/Néel temperatures, in accordance with the enhancement of the interlayer coupling in 


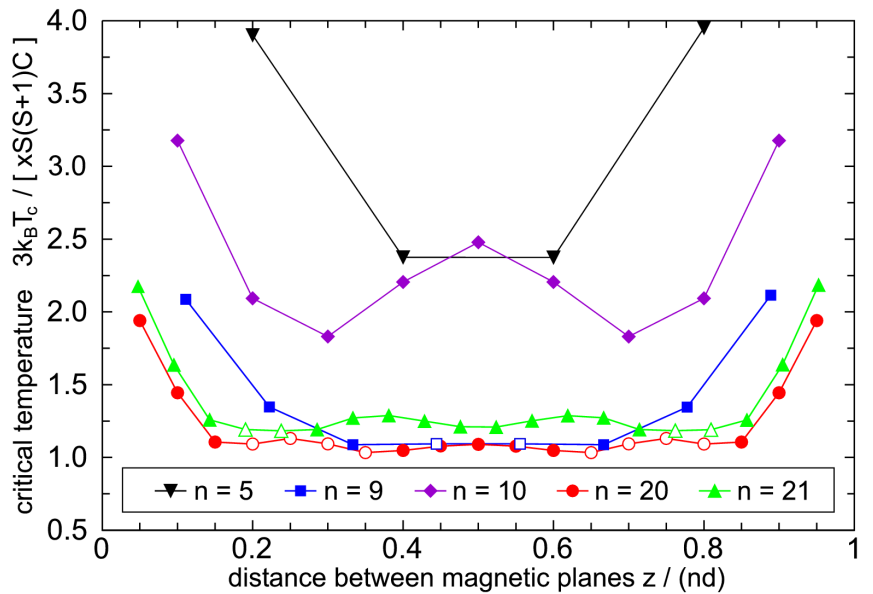

Fig. 3. The Curie and Néel temperatures vs. the distance between magnetic planes for several film thicknesses $n$.

Fig. 2. In particular, for $n=10$ a single maximum occurs for $z$ corresponding to half-thickness of the film, whereas for $n=21$ two symmetrical maxima appear. Such enhancement of the critical temperatures, oscillating with $n$, has the same origin as the interlayer coupling oscillations.

It can be concluded that the interlayer RKKY coupling in thin films shows an important dependence on the magnetic planes separation $z$, the film thickness $n$ and the charge carriers concentration $\rho$. With the help of $J(r, z)$, all relevant magnetic properties of the thin film can be obtained. The vital feature of these quantities in such a system is the existence of quantum size effects. In particular, they have been noticed in the critical Curie/Néel temperatures.

The presence of IMC coupling has been confirmed in the experimental studies of $\mathrm{Ga}_{1-x} \mathrm{Mn}_{x} \mathrm{As} / \mathrm{GaAs}$ or $\mathrm{Ga}_{1-x} \mathrm{Mn}_{x} \mathrm{As}_{2} \mathrm{Al}_{x} \mathrm{Ga}_{1-x} \mathrm{As}$ thin films and superlattices, for example by means of neutron scattering [13] or magnetization measurements [14]. The coupling strength varies with non-magnetic spacer thickness and vanishes either if the spacer is thick enough or the potential barrier in between the magnetically doped layers is created, preventing the charge carriers from penetrating this region. It is noticeable that only the ferromagnetic coupling has been detected so far. Interestingly, the dependence of the Curie temperature on the spacer thickness [15] was remarkably non-monotonous.

The theoretical approaches to the problem of IMC determination in diluted magnetic semiconductors (DMS) trilayers/superlattices of $\mathrm{Ga}_{1-x} \mathrm{Mn}_{x} \mathrm{As} / \mathrm{GaAs}$ include for example the work based on local spin density approximation for free carriers [16], where the oscillatory, RKKY-like coupling was predicted, indicating a noticeable antiferromagnetic range. Moreover, the studies employing the single parabolic band $k \cdot p$ approximation [17] focus on finding a distinct antiferromagnetic range and predict a similar kind of IMC behavior. On the other hand, the 
calculations in tight binding approximation for IV-VI semiconductor superlattices [18], result in rather monotonical dependence of IMC energy on the nonmagnetic spacer thickness, either ferro- or antiferromagnetic in sign, but concern very different substance.

In order to make connection with the experimental data, so far proving only the existence of ferromagnetic couplings, we emphasize the importance of two factors. Firstly, the spatial disorder-induced scattering of carriers, effectively leading to their localization and to damping of the long-range interaction, can be expected to impede the observation of antiferromagnetic IMC. Moreover, the up to now experimentally investigated Mn-doped layers in GaAs are at least a few monolayers thick, which results in some kind of averaging of the IMC interaction energy and can reduce the otherwise weak antiferromagnetic coupling. It should also be emphasized that our prediction of the antiferromagnetic coupling is limited to a rather narrow range of interplanar distances.

\section{Acknowledgments}

We gratefully acknowledge computational support on Hugo cluster at Department of Theoretical Physics and Astrophysics, P.J. Šafárik University in Košice.

\section{References}

[1] A.K. Bhattacharjee, C. Benoit à la Guillaume, Solid State Commun. 113, 17 (1999).

[2] J. Schliemann, J. König, A.H. MacDonald, Phys. Rev. B 64, 165201 (2001).

[3] L. Brey, G. Gómez-Santos, Phys. Rev. B 68, 115206 (2003).

[4] M.A. Boselli, A. Ghazali, I.C. da Cunha Lima, Phys. Rev. B 62, 8895 (2000).

[5] T. Balcerzak, in: Trends in Thin Solid Films Research, Ed. A.R. Jost, Nova Science Publishers, Inc., New York 2007, p. 249.

[6] T. Balcerzak, J. Magn. Magn. Mater. 310, 1651 (2007).

[7] M.A. Ruderman, C. Kittel, Phys. Rev. 96, 99 (1954); T. Kasuya, Prog. Theor. Phys. 16, 45 (1956); K. Yosida, Phys. Rev. 106, 893 (1957).

[8] C. Kittel, in: Solid State Physics, Vol. 22, Eds. F. Seitz, D. Turnbull, H. Ehrenreich, Academic Press, New York 1968, p. 1.

[9] Y. Yafet, Phys. Rev. B 36, 3948 (1987).

[10] M.T. Béal-Monod, Phys. Rev. B 36, 8835 (1987).

[11] D.N. Aristov, Phys. Rev. B 55, 8064 (1997).

[12] T. Balcerzak, Thin Solid Films 515, 2814 (2006).

[13] W. Szuszkiewicz, E. Dynowska, B. Hennion, F. Ott, M. Jouanne, J.F. Morhange, M. Karlsteen, J. Sadowski, Acta Phys. Pol. A 100, 335 (2001).

[14] N. Akiba, F. Matsukura, A. Shen, Y. Ohno, H. Ohno, A. Oiwa, S. Katsumoto, Y. Iye, Appl. Phys. Lett. 73, 2122 (1998). 
[15] R. Mathieu, P. Svedlindh, J. Sadowski, K. Światek, M. Karlsteen, J. Kanski, L. Ilver, Appl. Phys. Lett. 81, 3013 (2002).

[16] T. Jungwirth, W.A. Atkinson, B.H. Lee, A.H. MacDonald, Phys. Rev. B 59, 9818 (1999).

[17] A.D. Giddings, T. Jungwirth, B.L. Gallagher, Phys. Status Solidi C 3, 4070 (2006); arXiv.org:0802.0275.

[18] J. Blinowski, P. Kacman, Phys. Rev. B 64, 045302 (2001). 